\title{
Extracranial Artery Stenosis is Associated with Total MRI Burden of Cerebral Small Vessel Disease in ischaemic stroke patients
}

\section{Tao Lu; Jiahui Liang; Yonghong Chen; Yicong Chen; Fubing Ouyang; Weixian Huang; Jinsheng Zeng ${ }^{\dagger}$}

Department of Neurology, National Key Clinical Department and Key Discipline of Neurology, Guangdong Key Laboratory for Diagnosis and Treatment of Major Neurological Diseases, The First Affiliated Hospital, Sun Yat-sen University, Guangzhou 510080, China

† Corresponding Author

Background and Purpose Extracranial artery stenosis (ECAS) is related to individual markers of cerebral small vessel disease (cSVD). However, little has been reported on the association between ECAS and the total burden of CSVD as assessed by magnetic resonance imaging (MRI). The purpose of this study was to investigate the relationship between ECAS and total CSVD burden in patients with ischaemic stroke.

Methods We included consecutive patients who underwent colour Doppler ultrasonography and brain MRI for evaluation of acute stroke. The presence and total MRI burden of CSVD, including lacunar infarctions (LIs), white matter hyperintensities (WMHs), cerebral microbleeds (CMBs) and enlarged perivascular spaces (EPVS), were investigated. ECAS was classified by severity: absent or mild stenosis, moderate stenosis, severe stenosis, or occlusion. The relationships among ECAS and total MRI burden of cSVD were examined.

Results Two hundred and tween-one patients were included in this study. The mean age of the participants was $61 \pm 12$ years, and $75.6 \%$ of them were male. Hypertension, current smoking, hyperlipidaemia and diabetic mellitus were frequent among the patients $(67.4 \%, 45.7 \%, 43.9 \%$ and $36.7 \%$, respectively), while the other vascular risk factors including previous stroke/TIA and alcohol consumption were less frequent (19.0\% and $15.4 \%$, respectively). Significant differences in age, hypertension and ECAS were found among groups of patients with different total CSVD burden. KruskalWallis test and the post-hoc analysis showed that the severity of ECAS was higher in severe burden group than moderate burden group and absent to mild burden group respectively (Corrected $\mathrm{P}=0.003$ and 0.001 , respectively). Ordinal regression indicated that ECAS (odds ratio 1.76, 95\% $1.16-2.69$ ) was significantly and independently associated with total CSVD burden after adjustment for the other vascular risk factors.

Conclusions In this study, high levels of ECAS were associated with coexisting advanced cerebral CSVD in ischaemic stroke patients.

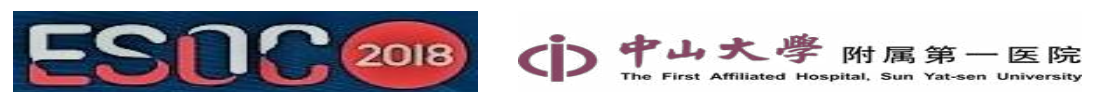

\begin{tabular}{|c|c|c|c|c|}
\hline & \multirow[b]{2}{*}{$\begin{array}{l}\text { All } \\
(\mathrm{n}=221)\end{array}$} & \multicolumn{3}{|c|}{ Total cSVD Burden } \\
\hline & & $\begin{array}{l}\text { Absent } \\
(\mathrm{n}=32)\end{array}$ & $\begin{array}{l}\text { mild } \\
(\mathrm{n}=105)\end{array}$ & $\begin{array}{l}\text { severe } \\
(n=84)\end{array}$ \\
\hline Age (years) & $61 \pm 12$ & $49 \pm 10^{*}$ & $60 \pm 10^{*}$ & $67 \pm 11^{*}$ \\
\hline Male, $n(\%)$ & $167(75.6)$ & $25(78.1)$ & $82(78.1)$ & $60(71.4)$ \\
\hline Hypertension, $\mathbf{n}(\%)$ & $149(67.4)$ & $16(50.0)^{*}$ & $67(63.8)^{\star}$ & $66(78.6)^{*}$ \\
\hline Diabetes mellitus, $\mathrm{n}(\%)$ & $81(36.7)$ & $11(34.4)$ & $40(38.1)$ & $30(35.7)$ \\
\hline Hyperlipidemia, $\mathbf{n}(\%)$ & $97(43.9)$ & $18(56.3)$ & $47(44.8)$ & $32(38.1)$ \\
\hline History of stroke/TIA, $\mathrm{n}(\%)$ & $42(19.0)$ & $3(9.4)$ & $19(18.1)$ & $20(23.8)$ \\
\hline Alcohol consumption, $\mathrm{n}(\%)$ & $34(15.4)$ & $5(15.6)$ & $18(17.1)$ & $11(13.1)$ \\
\hline Current smoking, $\mathbf{n}(\%)$ & $101(45.7)$ & $17(53.1)$ & $54(51.4)$ & $30(35.7)$ \\
\hline \multicolumn{5}{|c|}{ Extracranial carotid artery stenosis, $\mathbf{n}(\%)^{\star}$} \\
\hline absent or mild & $195(88.2)$ & $30(93.8)$ & 99(94.3) & $66(78.6)$ \\
\hline moderate & $8(3.6)$ & $1(2.7 \%)$ & $1(1.0)$ & $6(7.1)$ \\
\hline severe & $8(3.6)$ & 0 & $2(1.9)$ & $6(7.1)$ \\
\hline occlusion & $10(4.5)$ & $1(2.7 \%)$ & $3(2.9)$ & $6(7.1)$ \\
\hline
\end{tabular}
K Table 2 Associations with oSVD score and Vascular Factors in Multivariable Ordinal Regression Analysis 